\title{
¿Cómo se realiza la fiscalización de alimentos en Chile?
}

\author{
XIMENA RAIMANN T. ${ }^{1}$ \\ 1. Pediatra, Unidad de Nutrición, Hospital Luis Calvo Mackenna. Departamento de Pediatría y Cirugía Infantil Oriente, \\ Facultad de Medicina, Universidad de Chile.
}

¿Cómo sabemos que los alimentos que reciben nuestros niños son inocuos? ¿Cuál es el organismo encargado de fiscalizar la industria alimentaria? ¿Se hace control de los alimentos? ¿Cada cuánto tiempo? ¿Qué tan rigurosos son estos controles? Estas son algunas preguntas que nos surgieron después de lo acontecido con el suplemento alimenticio ADN® (Laboratorio B. Braun Medical), producto con el cual muchos de nosotros estábamos familiarizados en nuestro quehacer médico. Previo al descubrimiento del error en la composición del $\mathrm{ADN} \AA$, ¿dudábamos que, cualquiera fuese el producto, lo que estábamos indicando a nuestros niños no correspondía a lo que decía en su etiqueta?.

Como Rama de Nutrición de la Sociedad Chilena de Pediatría tratamos de dar respuesta a estas preguntas y nos dimos cuenta que no las teníamos todas, por lo que nos sentimos en la obligación de investigar al respecto para asegurar a la comunidad que lo que indicamos a nuestros pacientes es inocuo y adecuado en su composición, especialmente, si se trata de productos para niños.

Entonces, ¿cuál es el ente fiscalizador?.

En Chile, es el Ministerio de Salud (MINSAL) el encargado de fiscalizar a la industria productora de alimentos, para lo cual hoy en día cuenta con dos herramientas: el Reglamento Sanitario de los Alimentos (RSA) y el Plan Regional de Salud Pública (PRSP) en su componente "Inocuidad de los alimentos".
El Reglamento Sanitario de los Alimentos, Decreto No 977 de 1996 del Ministerio de Salud, detalla en sus 543 artículos los requisitos de higiene en la elaboración de los alimentos, las disposiciones generales de la rotulación, el uso de aditivos, las especificaciones microbiológicas, la reglamentación para cada grupo de alimentos y las sanciones, entre otros.

Dentro de los grupos de alimentos se encuentran los denominados "Alimentos para Regímenes Especiales", que son aquellos elaborados o preparados especialmente para satisfacer necesidades particulares de nutrición determinadas por condiciones físicas, fisiológicas o metabólicas específicas. En este grupo se encuentran las fórmulas para lactantes, las preparaciones comerciales de alimentos infantiles y los suplementos alimenticios. El ADN ${ }^{\circledR}$ está incluido dentro de esta denominación.

El PRSP se encarga de dar las orientaciones técnicas para el cumplimiento de metas de fiscalización a las regiones. Es la Autoridad Sanitaria Regional (ASR) o Secretaría Regional Ministerial (SEREMI) de Salud la que tiene la atribución legal de velar por la inocuidad de los alimentos que consume la población, para lo cual debe desarrollar acciones permanentes de control y vigilancia sanitaria de los procesos y los alimentos en toda la cadena de producción, elaboración, comercialización y consumo de estos.

La Organización Panamericana de la Salud, 
ha definido las Buenas Prácticas de Fabricación (BPF) y el Sistema de Análisis de Peligros y de Puntos Críticos de Control (HACCP: del inglés: Hazard Analysis and Critical Control Point) como los métodos modernos para el control de alimentos, a utilizar por parte de los gobiernos e industrias. Con la incorporación de estas herramientas, la industria sería el responsable primario de la inocuidad de los alimentos.

De acuerdo al artículo 69 del RSA, los establecimientos de producción, elaboración, preservación y envase de alimentos deben cumplir con las Buenas Prácticas de Fabricación (BPF), en forma sistematizada y auditable. Aquellos alimentos que la ASR determine tendrían que implementar la metodología HACCP, que es un sistema de seguridad que se basa en la prevención y brinda un método sistemático y acucioso para analizar los procesos realizados a los alimentos, define los peligros posibles y establece los puntos de control críticos, para evitar que lleguen al consumidor alimentos no seguros. La norma HACCP está basada en el Codex Alimentarius desarrollado por la Organización de las Naciones Unidas para la Agricultura y la Alimentación y la Organización Mundial de la Salud.

Con la adopción de metodologías como el HACCP, son las empresas las que se comprometen a diseñar sistemas de control de gestión sanitaria. Por otro lado, cambia el concepto del papel fiscalizador de los servicios oficiales, el cual se orienta a actividades de evaluación del cumplimiento de los compromisos establecidos por las empresas, cuando cuentan con un plan de HACCP. Los servicios oficiales realizan auditorías y verifican dichos cumplimientos.

Previo a la aplicación del sistema HACCP, es necesario que los establecimientos cuenten con una base sólida de las BPF, que son una herramienta de garantía de calidad.

Existe un sistema de control de higiene de los alimentos basado en la inspección de los establecimientos para verificar las condiciones estructurales y funcionales de los establecimientos, que tiene como base el riesgo epidemiológico de los alimentos, de modo que los establecimientos se dividen en cuatro categorías según el tipo de alimentos que producen y su riesgo asociado, por ejemplo productos que por su naturaleza son más susceptibles de contaminación o aquellos destinados a poblaciones más vulnerables. Cada SEREMI cuenta con un plan de inspección que determina la frecuencia de visitas de los establecimientos, lo que depende de la disponibilidad del recurso humano y el riesgo de los establecimientos. A modo de ejemplo se considera óptimo que un establecimiento de alto riesgo se visite al menos una vez al mes. Complementario a esto, existe un programa de vigilancia de la calidad sanitaria de los alimentos, que incluye toma de muestras para análisis microbiológicos y físicoquímicos.

Desde año 2007 como parte del PRSP y dentro de la estrategia de implementación del nuevo sistema de control de calidad, HACCP, se estableció como meta la observación de las BPF escritas y auditables en al menos el 30\% de los establecimientos de alto riesgo sanitario.

Queda claro que la periodicidad de los controles a la industria alimentaria depende del recurso humano, que es claramente insuficiente, ya que en nuestro país los fiscalizadores no sólo ven las industrias de alimentos, sino también restaurantes, hospitales, Ley de tabaco, rabia y otras zoonosis; control de las empresas para verificar salud ocupacional de sus trabajadores, saneamiento básico, agua potable y residuos sólidos, entre otros .

Con respecto a los alimentos importados, éstos son controlados también por la SEREMI. La autorización de su uso y consumo se realiza partida por partida, sobre la base de un sistema que incluye la inspección ocular y el análisis de laboratorio. La frecuencia e intensidad de estos controles varía en función del riesgo epidemiológico del alimento y de su historia sanitaria.

¿En qué etapa se encuentra la implementación del HACCP? La entrada en vigencia de la Resolución que determina la obligatoriedad, por parte de las empresas, de contar con planes de HACCP (Resolución Exenta $n^{\circ} 658$ de 2006), fue postergada para el año 2009 (resol. Ex. N ${ }^{\circ}$ 187 de 2008). En ella se determinaba el plazo para la implementación de HACCP de acuerdo a tres grupos prioritarios, conforme al riesgo sanitario y al nivel de ventas de las empresas. Dentro de los alimentos del grupo de primera prioridad están: los alimentos de uso infantil, la leche y productos lácteos, las carnes y los 
helados; en el grupo de segunda prioridad están, entre otros, los productos de pastelería y los platos preparados y, en el tercer grupo, los productos de confitería y los elaborados a partir de cereales. Así, las empresas "grandes" que fabrican alimentos que se encuentran en el grupo de Primera Prioridad deberían haber implementado el HACCP dieciocho meses después del decreto de 2006.

En la Resolución de HACCP, se establece que sin perjuicio de las auditorías internas (de responsabilidad del procesador) o externas (de responsabilidad de terceros) que se realicen, la auditoría externa oficial de los planes HACCP se realizará por la Autoridad Sanitaria Regional, la que queda incluida en sus actividades de vigilancia programada y que podrá certificar la conformidad a solicitud del interesado.

¿Qué rol juega el Instituto de Salud Pública (ISP)?. Es el laboratorio de referencia nacional para los análisis químicos y bromatológicos, depende del MINSAL y se encarga de analizar las muestras que envía la Autoridad Sanitaria, cuando ésta lo requiere.

A partir de esta revisión queda en evidencia que la autoridad sanitaria aún no es capaz de garantizar un proceso de fiscalización totalmente confiable; de la información existente se infiere que se debe agilizar la implementación de medidas de autocontrol de las empresas alimentarias (como el HACCP) y comprobar que este sea realizado con altos estándares técnicos y en forma permanente, aumentando el recurso humano para lograr una mayor frecuencia en los controles de los alimentos.

Después de lo acontecido con el ADN ${ }^{\circledR}$ se requiere con urgencia un control más periódico al grupo de alimentos para regímenes especiales, tomando en cuenta que muchas veces es la única alimentación que recibe el paciente, como es el caso de las fórmulas adaptadas en lactantes menores o las fórmulas a ser administradas por vía enteral en niños y adolescentes enfermos.

Como médicos pediatras también tenemos un papel que cumplir al respecto y debemos estar alerta a posibles dificultades con las herramientas que usamos para tratar a nuestros pacientes, sean éstas alimentos u otros como medicamentos, radioterapia o vacunas, pero en definitiva, es la autoridad y la industria alimentaria, en sus roles respectivos, los que deben asegurarnos que los alimentos que consumimos son confiables en su higiene y composición y por ende, que no pondrán en riesgo nuestra salud.

\section{Referencias}

1.- Reglamento Sanitario de los Alimentos. Decreto $\mathrm{N}^{\mathrm{o}}$ 977/96. Ministerio de Salud. República de Chile.

2.- Norma técnica para la determinación de implementación del Análisis de Peligros y de Puntos Críticos de Control (HACCP) en establecimientos de alimentos. Resolución exenta No 658 de 2006. Ministerio de Salud. República de Chile. 